\title{
Overview and Recent Accomplishments of the Advanced Mirror Technology Development (AMTD) for large aperture UVOIR space telescopes project
}

\author{
H. Philip Stahl \\ NASA Marshall Space Flight Center \\ SUBMITTED to: \\ SPIE Conference on \\ UV/Optical/IR Space Telescopes and Instrumentation, \\ San Diego CA \\ August 25-29, 2013
}

\begin{abstract}
Per Astro2010, a new, larger UVO telescope is needed to answer fundamental scientific questions, such as: is there life on Earth-like exoplanets; how galaxies assemble stellar populations; how baryonic matter interacts with intergalactic medium; and how solar systems form and evolve. And, present technology is not mature enough to affordably build and launch any potential UVO concept. Advanced Mirror Technology Development (AMTD) is a funded SAT project. Our objective is to mature to TRL-6 the critical technologies needed to produce 4-m or larger flight-qualified UVOIR mirrors by 2018 so that a viable mission can be considered by the 2020 Decadal Review.
\end{abstract}

AMTD uses a science-driven systems engineering approach. We mature technologies required to enable the highest priority science AND result in a high-performance low-cost low-risk system. To provide the science community with options, we are pursuing multiple technology paths. We have assembled an outstanding team from academia, industry, and government with extensive expertise in astrophysics and exoplanet characterization, and in the design/manufacture of monolithic and segmented space telescopes. One of our key accomplishments is that we have derived engineering specifications for advanced normal-incidence monolithic and segmented mirror systems needed to enable both general astrophysics and ultra-high contrast observations of exoplanets missions as a function of potential launch vehicle and its inherent mass and volume constraints.

We defined and initiated a program to mature 6 key technologies required to fabricate monolithic and segmented space mirrors. Some of our technical milestone accomplishments include:

- $\quad$ Large-Aperture, Low Areal Density, High Stiffness Mirror Substrates: We successfully demonstrate (on a $43 \mathrm{~cm}$ diameter cut-out of a 4 meter mirror) a new fabrication process (stacked core low-temperature fusion) which has extended the previous state of the art for deep core mirrors from less than $300 \mathrm{~mm}$ to greater than $400 \mathrm{~mm}$. This technology advance leads to stiffer 4 to 8 meter class monolithic primary mirror substrates at lower cost and risk.

- Support System: We have generated point designs for 4- to 8-m mirror support systems which minimize internal mirror stress using a new opto-mechanical design and modeling tool.

- Mid/High Spatial Frequency Figure Error: We successfully demonstrated the ability to produce a zerogravity optical surface of $<6 \mathrm{~nm}$ rms at a $2 \mathrm{C}$ operating temperature.

- Segment Edges: We successfully demonstrated an achromatic edge apodization process to minimize segment edge diffraction and straylight on high-contrast imaging PSF.

- Segment to Segment Gap Phasing: We investigated utility of correlated magnetic interfaces to reduce segment to segment co-phasing error. But determined that it cannot achieve the extreme $(<10 \mathrm{pm} \mathrm{rms})$ engineering tolerances needed to meet science requirements.

Integrated Model Validation: We developed a new opto-mechanical design and modeling tool which creates point designs of monolithic and segmented primary mirrors $>10 \mathrm{X}$ faster than commercial tools and facilitates transfer of a high-resolution mesh to various mechanical and thermal analysis tools. We conducted trade studies for 4-m and 8-m mirror systems; created and validated models to predict gravity sag and 2C thermal gradients; defined and started implemented a systems analysis framework that combines simulation results from multiple engineering disciplines to predict on-orbit optical perfo 